\title{
Contig 4
}

\section{Contig 5}

PCR for gap closing $\longrightarrow \longrightarrow$

ap size approx.

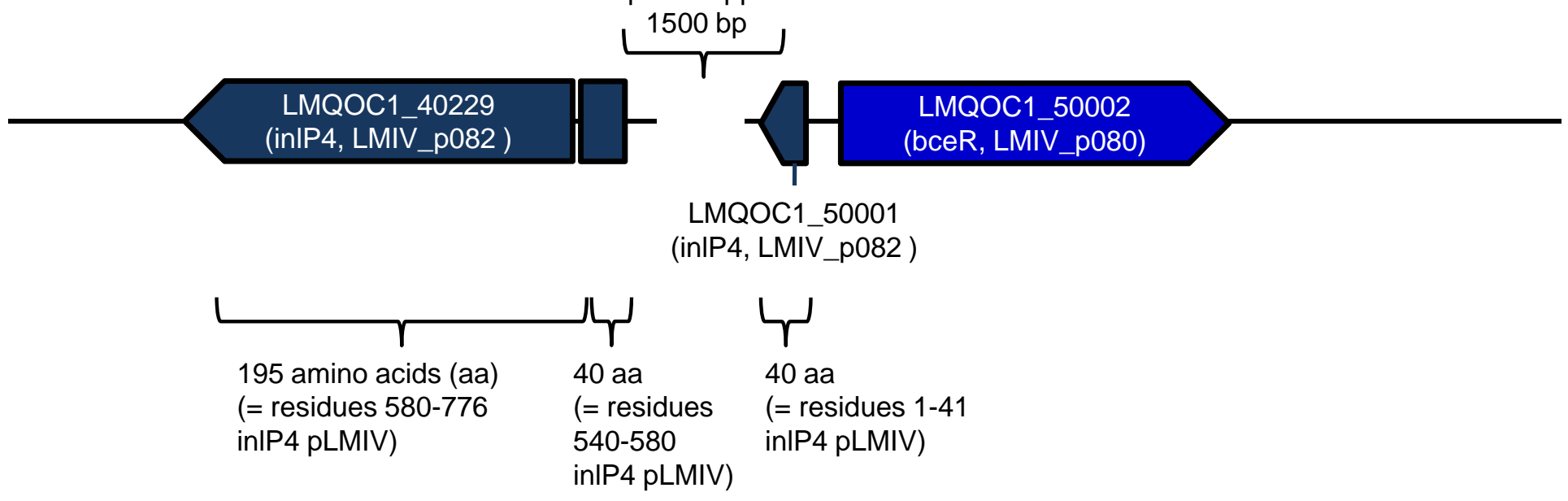

Figure S6: Genomic region surrounding the gap between contig 4 and contig 5 in the L. monocytogenes QOC1 genome. 